\title{
Hearing loss as a newly recognized symptom of GSD type I. A clinical report of four unrelated Polish patients.
}

Katarzyna Iwanicka-Pronicka ( $\nabla$ k.iwanicka-pronicka@ipczd.pl )

Instytut Pomnik -Centrum Zdrowia Dziecka https://orcid.org/0000-0003-2164-6663

Joanna Trubicka

Instytut Pomnik -Centrum Zdrowia Dziecka

Maciej Pronicki

Instytut "Pomnik-Centrum Zdrowia Dziecka"

Edyta Szymanska

Instytut Pomnik -Centrum Zdrowia Dziecka

Elżbieta Ciara

Instytut Pomnik -Centrum Zdrowia Dziecka

Dariusz Rokicki

Instytut Pomnik -Centrum Zdrowia Dziecka

\section{Saskia Wortmann}

Technishe UniversitatMunchen

\section{Research}

Keywords: GSDla, GSDIb, hearing loss, ski-slope audiogram, SLC37A4, G6PC

Posted Date: June 2nd, 2020

DOI: https://doi.org/10.21203/rs.3.rs-31974/v1

License: (9) (i) This work is licensed under a Creative Commons Attribution 4.0 International License.

Read Full License 


\section{Abstract}

Background: Glycogen storage disease (GSD) type la and Ib is the most severe type of all hepatic GSDs. It presents with serious liver and metabolic complications, as well as in type lb with severe infections due to neutropenia. So far, the hearing impairment has not been reported in these patients. Hearing status was assessed in a group counting 40 probands with GSDI (20 patients with each subtype). Hearing loss was detected in four patients. They did not pass newborn hearing screening. Due to the development of severe metabolic complications in infancy, audiological diagnosis was postponed until the early school age, when speech and language delay was striking.

Hearing tests revealed the bilateral sensorineural hearing impairment with the audiograms presenting skislope curves in all patients. Next-generation sequencing (NGS) using gene panel: TruSight OneTM Sequencing Panel Kit (Illumina, San Diego, CA, USA) or whole-exome sequencing (WES) using SureSelect Human All Exon Kit (Agilent, 60mb V6) were performed in order to find possible genetic background of auditory dysfunctions in these patients.

Results: Hearing impairment seems to affect about10\% GSDI patients. Molecular analysishas revealed the common homozygous pathogenic variant in G6PC in two GSDla probands. Common pathogenic variant in SLC37A4 gene was identifiedin one GSDIb patient, whereas the second case was found to be a carrier of one common and one rare pathogenic variant in SLC37A4 gene (in trans ). No change in the genes involved in auditory pathway dysfunction was found, although the patients displayed the same audiological characteristics.

Conclusions: Sensorineural hearing lossappears to be a clinical complication of GSDI encountered in approximately one out of ten cases. Carefulmonitoring of auditory functions is recommended among GSDI patients, especially in prelingulal period. Appropriate early detection and intervention (fitting with hearing aids) prevents potential speech and language impairment.

\section{Background}

Glycogen storage disease (GSD) is an inborn error of carbohydrates metabolism, which includes 8 liver types with hypoglycemia as an underlying symptom [1,2]. Among them, type I with la and Ib subtypes (with neutropenia), is the most severe one. It is an autosomal recessive condition in case of subtype la

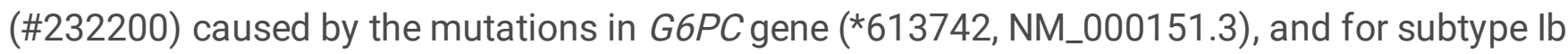
(\#232220) in SLC37A4 gene ( ${ }^{\left.* 602671, N M \_001164277.1\right) . ~ B o t h ~ t y p e s ~ a r e ~ c h a r a c t e r i z e d ~ b y ~ i n a b i l i t y ~ t o ~}$ convert glucose-6-fosfate to glucose due to either glucoso-6-fosfatase deficiency (type la) or an impaired transport of the enzyme to the microsomes caused by a deficiency of translocase glucoso-6-fosfatase (type lb) [3]. This leads to massive glycogen accumulation in the liver. So far, no strong genotypephenotype correlations have been identified for this disorder $[4,5]$.

GSDI presents with various hepatic and metabolic complications such as: hypertransaminasemia, hyperlipidemia, hyperurykemia, lactic acidemia, growth retardation and increased risk of developing liver 
adenomas [6, 7]. Neutropenia causing serious infections and inflammatory bowel disease is an additional complication in type lb [8].

In this type, both gluconeogenesis and glycogenolysis are impaired, thus patients present with most severe hypoglycemia which occurs as soon as $2-4$ hours after the meal. Since ketogenesis is poor in this type, children may develop seizures. However, neither intellectual nor neurological complications have been firmly associated with GSDI, and sporadic cases which have been published were found to correlate with the frequency of hypoglycemic episodes, particularly in patients with poor dietary conditions [9]. Hearing impairment seems to be unrecognized symptom in any subtype of GSDI.

\section{Results}

All 40 patients with GSDI underwent a panel of hearing tests. Normal hearing was confirmed in 36 subjects. Sensorineural hearing impairment was detected in four probands (10\%). SNHL displayed similar audiological characteristics in our subjects. Audiometric curves showed a ski-slope pattern. Despite appropriate discrimination of low frequencies sounds, patients could not hear mid-range and high tones. Speech detection was poor in all cases. All patients required devices improving hearing quality.

\section{Results of the molecular investigation}

Molecular analysis revealed the common homozygous pathogenic variant c.247C>T (p.Arg83Cys) in G6PC in two GSDla probands (Patients 1 and 2). Compound heterozygosity status of one common mutation c.1042_1043delCT (p.Leu348fs) and one novel pathogenic variant c.341A>G (p.Gln114Arg) in SLC37A4 gene was detected in Patients 3. Patient 4 was found to be a carrier of common homozygous pathogenic variant in SLC37A4 gene (Table 1). Sanger sequencing confirmed the results of the NGS analysis. Parental studies confirmed heterozygosity status and in trans allelic pattern of inheritance for identified variants in all four cases. Molecular evaluation included also screening of pathogenic variants in known 212 deafness causing genes included in TruSight One panel or 262 known genes encompassed by whole exome. The analysis disclosed only likely pathogenic variant c.542C $>\mathrm{T}$ p.(Thr181Met) of CLCN7 gene (NM_001287.5) in the DNA of the Patient 2 and her father. 


\begin{tabular}{|l|l|l|l|l|}
\hline Patient & Patient 1 & Patient 2 & Patient 3 & Patient 4 \\
\hline GSDI & Type IA & Type IA & Type IB & Type IB \\
\hline Gene & G6PC & G6PC & SLC37A4 & SLC37A4 \\
\hline Reference sequence & NM_000151.4 & NM_000151.4 & NM_001164278.2 & NM_001164278.2 \\
\hline Molecular variant on allele 1 & c.247C>T & c.247C>T & c.1108_1109delCT & c.1108_1109delCT \\
\hline Molecular variant on allele 2 & c.247C>T & c.247C>T & c.341A>G & c.1108_1109delCT \\
\hline Protein effect on allel 1 & p.(Arg83Cys) & p.(Arg83Cys) & p.(Leu370ValfsTer53) & p.(Leu370ValfsTer53) \\
\hline Protein effect on allel 2 & p.(Arg83Cys) & p.(Arg83Cys) & p.(Gln114Arg) & p.(Leu370ValfsTer53) \\
\hline
\end{tabular}

\section{Discussion}

GSDI is an inborn error of metabolism caused by the deficiency of glucose-6-phosphatase enzyme or its translocase through the mitochondrial membrane. This metabolic block impairs the ability of the liver to produce free glucose from glycogen and from gluconeogenesis [1]. Children with GSDI present with hepatomegaly, growth retardation, lactic acidosis, hyperuricemia, hyperlipidemia, hypertriglyceridemia, and/or hypoglycemic seizures $[2,4]$. In addition to that, subtype $\mathrm{lb}$ is associated with chronic neutropenia and impaired neutrophil and monocyte function, which results in recurrent bacterial infections, and oral or intestinal mucosal ulcers [8]. Neutropenia and recurrent infections of upper respiratory tract, tonsillitis, sinusitis and otitis seem to be potential causes of auditory complications in GSDIb patients. Hearing loss is a rare complication in GSDI patients, however it may be secondary to the middle ear damage, thus revealed a conductive, potentially reversible character [10].

Although these patients may experience hypoglycemic seizures with poor ketones such episodes do not seem to have firm negative neurological impact, since neither intellectual nor sensory perception disorders have been associated with GSDI [4]. Any changes in IQ, MRI findings or EEG, reported in these patients, were found to correlate with the frequency of hypoglycemic episodes, particularly in those with poor dietary compliance $[9,11]$.

We present four unrelated GSDI patients with hearing loss. Similar audio-profile of our probands is striking. They display non-progressive, sensorineural impairment of cochlear origin, with slopping-down pattern of audiometric curves. The diagnosis of SNHL based on the battery of hearing tests was performed only in the early school age. However, strong evidence indicates congenital origin of hearing impairment: all four children did not pass newborn hearing screening tests, then displayed delayed speech development.

Schuknecht and Gacek studied the histopathology of the inner ears of patients with SNHL [12]. They distinguished four types of SNHL: sensory, neural, metabolic and cochlear, basing on a correlation between the shape of audiogram and impaired structures. The ski-slope curves associated with isolated 
hair cells damage were called the sensory type. This was observed among our GSDI probands. The same audiometric pattern was widely observed in SNHL patients being the m.1555A > G mutation carriers [13].

Since the impairment involves mid-range and high frequencies, normal hearing at low frequencies has allowed some speech development, though with a delay. Patients were referred to audiologist mainly due to speech delay, defects in articulation and specific learning difficulties.

Early occurrence of GSDI symptoms postponed audiological verification, that was performed after their discharge from neonatal wards. Following the late diagnosis of SNHL, the patients were fitted with hearing aids, however, cochlear implant surgery was strongly recommended for two patients with both types of GSDI. Despite the obvious impact of cochlear implant surgery on auditory benefits and educational success, delayed implantation procedure does not provide satisfactory compensation of profound auditory dysfunction and speech deficits.

There is available only one case of Iranian GSDIb patient with severe hearing loss [11]. The authors did not provide any audiological details, however they assumed possible relationship between hearing impairment and repeated severe hypoglycemic convulsions.

The exact pathomechanism of hearing impairment in GSDI is not known so far, and why it is such a rare complication. We have not found any literature describing histopathological findings of the inner ear of patients with GSDI, therefore it is hard to speculate of possible glycogen accumulation in the inner ear structures. One theory showed probable ototoxic effect of recurrent hypoglycemia, dyslipidemia or hypertension $[9,11]$. Mangabeira-Albernaz described mild, fluctuating, reversible hearing loss in patients with disturbed carbohydrate metabolism and hyperinsulinemia [14]. Another widely postulated hypothesis presents the impact of oxidative stress and free radicals on a destruction of hair cells [15], Prasad suggested the crucial role of microRNA [16]. Kondo presented interesting results of the study on the influence of fructo-oligosaccharides, acting as prebiotic, on a development of hearing loss [17]. He observed an increased level of short chain fatty acids after fructo-oligosaccharides ingestion and postulated its influence on overexpression of molecules detected in the inner ear, especially: short chains fatty acids receptor, neurotrofin, tyrosine kinase receptor, brain derived neurotropic factor and its receptor. He suggested a damaging effect of all these molecules on hair cells. The potential negative impact of GSDI dietary treatment with long-term supplementation of corn-starch may on hair cells required case studies for verification.

The only glycogenosis with occasional auditory dysfunction, reported in literature, is Pompe disease (GSDII) [18]. However, Pompe disease is a lysosomal storage disease with wide range of muscular manifestations [19]. The mechanism of hearing loss in Pompe disease is due to stapedial muscle involvement, and was demonstrated in adult patients with this disease [20]. Another study by an Italian group showed that over $50 \%$ of ears of 20 patients with GSDII had a hearing impairment, and $57 \%$ of them was a sensorineural deficit [18]. Another studies supported this data [18, 20]. A group from Rotterdam demonstrated in a knockout mouse model of Pompe's disease the storage of glycogen in the inner ear structures (hair cells of the cochlea, the supporting cells, the stria vascularis, and the spiral 
ganglion cells), which has proved that the cochlear pathology is the most likely cause of hearing loss in infantile GSDII [18].

In order to find other potential genetic background of hearing loss in patients with GSDI, a molecular testing using NGS was performed. Dominant pathogenic variant c.542C > T of the $C L C N 7$ gene was detected in Patient 2 and her healthy father. This change is related to osteopetrosis type 2 (\#166600), the entity called Albers Schonberg disease. This is a congenital malformation of bones resulting in increased bone density. Short stature and frequent fractures are the major features. Hearing loss may occur in osteopetrosis type 2, however its onset is generally after the second decade of life. Hearing deterioration is usually a secondary finding to osteopetrotic malformation of the temporal bone [21]. Neither our proband nor her father meet the phenotypic criteria of osteopetrosis type 2. Congenital origin of hearing loss in our proband, no middle ear alterations, no past history of otitis exclude the hypothesis of pathogenic role of the c.542C > T variant in CLCN7 in the etiology of hearing loss. No other pathogenic variant in hearing loss related genes was detected.

This issue enables to hypothesis that hearing impairment may be a new symptom of GSDI. Although the pathomechanism of hearing disorders in individuals with GSD type I remains unknown, our results emphasize the need for monitoring of auditory functions in these patients, in order to prevent potential speech and language impairment, with early implementation of hearing aids.

\section{Conclusions}

Congenital, non-progressive, sensorineural hearing impairment is a rare symptom of GSD type I, that can be diagnosed equally often in patients suffering from GSDla and GSDIb. Audiometric curves show slopping-down shape in all subjects. Patients with this type of hearing impairment correctly discriminate low-frequency sounds, that allow them to develop a certain level of spoken language even without hearing-improving devices. However, early fitting with hearing aids or cochlear implantation enables proper language and speech development. The pathomechanism of hearing loss in GSDI patients remains unclear. Children with inborn errors of metabolism who did not pass newborn hearing screening test require immediate audiological verification. Careful monitoring of hearing functions in GSDI patients, especially in prelingual period, is highly recommended.

\section{Methods}

\section{The aim, design and setting of the study}

Sensorineural hearing loss (SNHL) was detected in four patients with GSD type I. The proper audiological diagnosis was postponed for each subject due to the development of the serious metabolic complications. Delayed speech development was noticed in all probands. Although speech therapy was implemented (for each child), no satisfactory improvement was observed. Therefore, the hearing tests were performed. Pure tone audiometry (PTA) revealed the ski-slope audiometric curves in all individuals. 
Although the diagnosis of hearing impairment was established at an early school age, the results of the newborn hearing screening indicating needs of audiological management and the speech impairment strongly suggest a pre-lingual origin of the hearing loss in our probands. Considering a similar pattern of the hearing tests results, together with the common inborn error of metabolism, molecular investigations were performed. Simultaneously, we assessed hearing status in the whole group of patients with GSD type I

\section{The group of patients with GSD type I}

The study group included 40 Polish patients with GSD type I diagnosed and treated in the Children's Memorial Health Institute in Warsaw, Poland. The group comprises 20 cases with subtype la and 20 subjects with subtype $\mathrm{lb}$. The median age of probands was 12 years, range: $5-40$ years. They all underwent audiological testing.

\section{The characteristics of four patients with GDSI and hearing impairment:}

Patient $1(\mathrm{MB})$

The first patient is a 14-year-old boy with GSDla. His general health status was stable due to corn starch dietary supplementation. The main health problems were hypertension (treated with angiotensinconverting-enzyme inhibitor), hepatomegaly, and persistent alterations in the results of the laboratory tests: lactic acidosis, hyperuricamia, hypercholesterolemia. Delayed speech development was noticed. Appropriate intensive therapy was introduced at the age of 5 years due to speech illegibility. He has followed the mouth of the speaker, needed repetition and required loud TV sounds. At school he had many problems, especially with understanding teacher's explanations and dictation.

A panel of hearing tests performed at the age of 8 displayed sensorineural hearing loss of the cochlear origin. Ski-slope shape of the audiogram meeting the criteria of partial deafness was revealed in PTA (Figure 1). The normal hearing threshold was observed only at low frequencies $(125 \mathrm{~Hz}-500 \mathrm{~Hz})$ in the better hearing ear, the hearing level at the mid-range and high frequencies $(1 \mathrm{kHz}-8 \mathrm{kHz})$ was below $90 \mathrm{dBHL}$. Type A tympanograms were registered in both ears. Neither distortion products otoacoustic emissions (DPOAE) nor stapedial reflexes above $500 \mathrm{~Hz}$ were registered. Brainstem evoked auditory potentials (BEAP) revealed profound hearing loss, which was consistent with the audiogram. Patient 1 was fitted with hearing aids, however, no satisfactory outcome was achieved, as suspected. A cochlear implant was recommended and remains under consideration due to the lack of the patient's acceptance of the method.

Patient 2 (AS)

The second patient is a 12 years-old girl suffering from GSDla. She was born at term in $38 \mathrm{Hbd}$ by CC (due to vaginal bleeding) after uneventful pregnancy. The birth weight was $3400 \mathrm{~g}$ and she was assessed at 10 points according to the Apgar score. 
At the 4. month of age an increasing lost of appetite accompanied by enlarged abdomen circumference and hepatomegaly was noticed. Laboratory tests revealed asymptomatic hypoglycaemia, hypertriglyceridemia, lactic acidosis and an increased level of liver function tests with normal bilirubin level and appropriate parameters of the clotting system. Liver core biopsy showed diffuse, non-zonal, predominantly macrovacuolar liver steatosis of moderate-severe degree, extending approximately $70-80 \%$ of the parenchyma. Mild degree of portal fibrosis without fibrous bridging were seen. There were no signs of inflammation, cholestasis, neither pathology of intrahepatic bile ducts. (Figure 2).

Following the diagnosis, an immediate dietary treatment for GSDla was commenced when she was 7 months of age. Her development was satisfactory. The main health problems were hypertension treated with angiotensin-converting-enzyme inhibitor (Tritace) and hepatomegaly accompanied with abdominal obesity. Lactic acidosis was a single blood marker of GSDla. She started speech therapy due to an inappropriate articulation when she was five. No improvements were observed. Subsequently, she encountered school problems with a hearing synthesis, learning to read, writing and understanding details. For those reasons she was referred to an audiology unit when she was 7 years old. A battery of hearing tests was performed. PTA revealed the ski-slope hearing curve. The audiogram displayed normal hearing at low and mid-range frequencies, with a sloping curve from $1 \mathrm{kHz}$ to high frequencies - up to the level of $90 \mathrm{~dB}$ (Figure 1).

Type A tympanograms were registered in both ears, stapedial reflexes were registered in all examined frequencies but $4 \mathrm{kHz}$. DPOAE were registered only below the $1500 \mathrm{~Hz}$. BEAP confirmed the audiometric hearing thresholds and the cochlear origin of the hearing impairment. Patient 2 was fitted with hearing aids and since then she enjoys auditory benefits.

\section{Patient 3 (SK)}

The third patient is a 9-year-old boy suffering from GSDIb. He was the first child of unrelated parents. He was born through natural delivery at $34 \mathrm{Hbd}$, the birth weight was $1870 \mathrm{~g}$, he obtained 8 points according to the Apgar score. The pregnancy was complicated by maternal diabetes mellitus. Recurrent upper respiratory tract infections, accompanied by episodes of severe hypoglycaemia, leukocytosis with neutropenia were noticed from the first months of age. The diagnosis of GSDIb was established at the 9 . month of life and subsequent dietary therapy was introduced. Patient 3 has got many health problems including hypertension, hepatomegaly and hypertrophic cardiomyopathy. Right kidney surgery was preformed when he was seven. A tumoral mass clinically suspected for abscess was detected. Microscopically renal parenchyma showed complete effacement of architecture by non-specific, polymorphic, partly purulent inflammatory infiltrate with interstitial fibrosis. Only a few glomeruli and tubules were preserved and discernible in fibro-inflammatory mass (Figure 3). Alterations detected in the results of blood tests of Patient 3 included hypertransaminasemia, lactic acidosis, hyperuricemia, hypercholesterolemia, and hypertriglyceridemia. He was persistently treated with granulocyte colonystimulating factor (G-CSF) - Neupogen due to leukopenia. Low white blood cells count was the key factor of recurrent infections, especially the recurrent otitis media. He was under ENT care from infancy. Hearing 
tests have been performed since he was 7 months. Both tympanometry and DPOAE tests indicated otitis media and BEAP confirmed hearing loss. Hearing aids were contradicted due to subsequent effusions from ears. A delayed speech development was observed. Adenoidectomy with insertion of the ventilation tubes into the tympanic membranes, equalizing pressure in the middle ear, was done when he was four years old. Audiological tests (BEAP, DPOAE, and tonal play audiometry) performed after surgery, displayed severe hearing impairment, while type B tympanograms confirmed the correct function of the tubes. Mastoidectomy of the left ear followed subsequent ear infections. PTA was done at the age of 5 years, the audiogram had a ski-slope shape, which was slightly improved in comparison to the previous ones (Figure 1). The normal hearing remained at low and the midrange frequencies $125 \mathrm{~Hz}-1500 \mathrm{~Hz}$ in the better hearing ear (the right ear). Mild sensorineural hearing impairment was found at mid-frequencies, and severe hearing loss was detected below $4 \mathrm{kHz}$ (at high frequencies). He was finally successfully fitted with hearing aids.

Patient 4 (MR)

The fourth patient with GSDIb associated with SNHL is already a 23-year-old man. His main health problems are short stature, hepatomegaly, glaucoma of the left eye, total retinal detachment of the left eye, and exotropia of the left eye. He underwent vitro-retinal surgery of the left eye.

He was born at term through spontaneous vaginal delivery, after uneventful pregnancy, with $3200 \mathrm{~g}$ and 10 points of the Apgar score. Neonatal adaptation problems with hyperbilirubinemia and hypoglycaemia were thought to be related to his intrauterine infection. Hypoglycaemia, hypertriglyceridemia, hypercholesterolemia, hypertransaminasemia, hyperuricemia, lactic acidosis and leukopenia have been reported since he was 3 months old. The diagnosis and dietary treatment of GSD1b was established at the age of 9 months. At the age of 6.5 years sensorineural hearing loss was detected based on PTA. The audiogram presented the ski-slope audiometric curve. Normal hearing was observed only up to $250 \mathrm{~Hz}$, severe impairment was detected already at mid-range frequencies and deafness above the $4 \mathrm{kHz}$ (Figure 1). He was fitted with hearing aids, immediately after the diagnosis, but no satisfactory speech improvement was observed. He was referred to a cochlear implant surgery.

\section{Audiological examinations}

Routine videootoscopy and following audiological tests were performed in all examined patients. Hearing threshold level was assessed with PTA (Interacoustics, AC40). The clinical interpretation of the degree of hearing impairment was based on ANSI (American National Standards Institute) and ISO (International Standards Organization) standards. The inner ear functions were checked with clinical DPOAE (Interacoustics, Titan). Middle ear functions were assessed with tympanometry and stapedial muscle reflexes (Interacoustics, AT235H).

The auditory pathways in the brainstem were checked with BEAP (GSI, Audera) in the four probands with hearing impairment. Chirp stimulus was used for detection of high frequencies $(2-4 \mathrm{kHz})$ and tone-burst 
stimuli to examine tones of $500 \mathrm{~Hz}$ and $1 \mathrm{kHz}$, respectively. BEAP were used to confirm the diagnosis of hearing impairment.

\section{Molecular investigation}

Genetic analyses were performed after obtaining informed consent from the Patient 4 and the parents of Patients 1-3. Genomic DNA was extracted by automated method (MagnaPure, Roche) or manual (phenolchloroform) method from peripheral blood samples of the probands and relatives. Next-generation sequencing (NGS) of Trusight One sequencing panel, created by Illumina for the simultaneous sequencing of 4,813 clinically relevant genes was used as a genetic target. NGS was performed on a HiSeq 1500 platform using an exome enrichment kit (Illumina) according to the manufacturer's protocol. Generated reads were first merged and low-quality reads removed. Then the reads were aligned to the hg19/hg38 (GRCh37/GRCh38) reference human genome. Potential PCR duplicates and reads mapping to multiple genomic locations were removed. In variant calling, any call with the ratio smaller than 0.2 was assumed to be homozygous, and the rest - heterozygous. Alignments were viewed with Integrative Genomics Viewer v. 2.3.82 (7). The detected variants were annotated using Annovar and converted to MS Access format for final manual analysis.

All the non-coding and common variants (minor allele frequency (MAF) above 0.01 in the general population) were discarded. The rare variants affecting the coding regions were filtered basing on the autosomal recessive mode of inheritance and predicted consequences at the transcript level. The variants were prioritized according to the population frequency and the predicted effect on the protein. The potential consequences were defined according to the conservation of the affected amino acids and in silico predictions by using different algorithms.

To identify the molecular basis of the glycogen storage disease, first we analyzed GSDI related genes. Additionally, in order to detect sensorineural hearing impairment potentially causative variants from TruSight One target, we applied a computational algorithm termed Phenotypic Interpretation of eXomes (PhenIX) [22]. The software evaluates and ranks detected gene variants based on pathogenicity and semantic similarity of hearing loss described by Human Phenotype Ontology (HPO) terms to those of known Mendelian diseases.

Additionally, for Patients 2 and 3 as well as for their parents, the whole exome analysis of case-parents' trios (trio-WES) using SureSelect Human All Exon Kit (Agilent, 60mb V6) were performed. The prepared libraries were subsequently sequenced as 100 bp paired - end reads on the Illumina Hiseq 4000 (trio-WES) to an average 141 -fold coverage with more than $98 \%$ of target sequences being covered over 20 -fold.

NGS data were analyzed using an in-house procedure, described in detail previously [23]. Additionally, for WES data, the allele frequency was assessed in an in-house database (Munich-Exome-Server which include data from 6,000 exomes). 
The interpretation of identified variants was based also on information reported in public databases: Human Gene Mutation Database (HGMD), Decipher, ClinVar, Online Mendelian Inheritance in Man (OMIM) and Varsome The Human Genomics Community.

The candidate pathogenic variants were verified in the probands and relatives, by Sanger sequencing using BigDye Chemistry (Applied Biosystems).

\section{Abbreviations}

GSDI - glycogen storage disease type I; GSDII - glycogen storage disease type II (Pompe disease); NGS next-generation sequencing; WES - whole-exome sequencing; SNHL - sensorineural hearing loss; PTA pure tone audiometry; ANSI - American National Standards Institute; ISO - International Standards Organization; dB HL - decibel hearing level; $\mathrm{Hz}$ - Hertz; DPOAE - distortion products otoacoustic emissions; BEAP - brainstem evoked auditory potentials; IQ - Intelligence Quotient, MRI - magnetic resonance imaging; EEG - electroencephalography; TV - television; CC - caesarian section; Hbd (lat. Hebdomas) - week of pregnancy; G-CSF - granulocyte colony-stimulating factor; ENT - ears nose throat; hg19/hg38 - human genome (reference); PCR - polymerase chain reaction; MAF - minor allele frequency; HPO - Human Phenotype Ontology; HGMD - Human Gene Mutation Database; OMIM - Online Mendelian Inheritance in Man.

\section{Declarations}

\section{Ethics approval and consent to participate}

The study was conducted according to the principles of the Helsinki Convention. The Bioethics Committee of the Children's Memorial Health Institute approved the protocol of the study.

\section{Consent for publication}

Participants signed consent for publication

\section{Availability of data and materials}

The datasets used and analyzed during the current study are available from the corresponding author on reasonable request

\section{Authors declare no competing interests}

\section{Funding}

No external funding was used for this study

\section{Authors' contribution:}


Katarzyna Iwanicka-Pronicka - concept and design of the study, audiological consultations, interpretation of the results of hearing tests, wrote the text of the manuscript

Joanna Trubicka - interpretation and molecular analysis of the TruSight One data, wrote a molecular part of the manuscript

Maciej Pronicki - histopatological assessment, preparing Figures

Elżbieta Ciara - interpretation and molecular analysis of TruSight One data, correction of the manuscript

Edyta Szymanska - interpretation of clinical data, correction of the manuscript

Dariusz Rokicki - interpretation of clinical data, consultations

Saskia Wortmann - interpretation and molecular analysis of WES data, critical correction of the manuscript

Acknowledgements: The authors acknowledge Mrs Swietlana Brzezinska, a secretary of the Department of Audiology and Phoniatrics for the preparation of the Figure 1

\section{References}

1. Fernandes J, Smit GPA. The Glycogen Storage Disease. Inborn Metabolic Diseases, 3rd rev Edn. Springer, Berlin 2000: 86-101.

2. Raghuveer TS, Gardg U. Inborn Errors of Metabolism in Infancy and Early Childhood: An Update. Am Fam Physician. 2006 (11):1981-1990.

3. Chou JY, Matern D, Mansfield BC, Chen YT. Type I glycogen storage diseases: disorders of the glucose-6-phosphatase complex. Curr Mol Med. 2002;2:121-43.

4. Kishnani PS, Austin SL, Abdenur JE, et al. Diagnosis and management of glycogen storage disease type I: a practice guideline of the American College of Medical Genetics and Genomics. Genet Med. 2014;16:e1.

5. Eminoglu TF, Ezgu FS, Hasanoglu A, Tumer L. Rapid screening of 12 common mutations in Turkish GSD 1a patients using electronic DNA microarray. Gene. 2013;518:346-50.

6. Banka S, Newman WG. A clinical and molecular review of ubiquitous glucose-6-phosphatase deficiency caused by G6PC3 mutations. Orphanet J Rare Dis. 2013 Jun;13:8:84.

7. Lei KJ, Chen H, Pan CJ, et al. Glucose-6-phosphatase dependent substrate transport in the glycogen storage disease type-1a mouse. Nat Genet. 1996;13:203-9.

8. Visser G, de Jager W, Verhagen LP, et al. Survival, but not maturation, is affected in neutrophil progenitors from GSD-1b patients. J Inherit Metab Dis. 2012;35:287-300.

9. Melis D, Parenti G, Della Casa R, et al. Brain damage in glycogen storage disease type I. J Pediatr. 2004;144:637-4.

Page $12 / 16$ 
10. Tommaso AMA, Hessel G, Gut Riccetto A, Semenzati GO, Gusmao RJ. Pre and post-operative otorhinolaryngology surgery care in patients with Glycogen Storage Disease type 1. Rev Paul Pediatr. 2019;37(4):516-9. doi:10.1590/1984-0462/;2019;37;4,00005.

11. Eghbali M, AbiriM, Talebi S, Noroozi Z, Shakiba M, Rostami P, et al. Genotype-phenotype correlation and description of two novel mutations in Iranian patients with glycogen storage disease $1 \mathrm{~b}$ Orphan. J Rare Dis. 2020;15:35. doi:10.1186/s13023-019-1266-3.

12. Shuknecht HF, Gacek MR. Cochlear pathology in presbyacousis. Ann Otol Rhinol Laryngol. 1993;102(1):1-16.

13. Iwanicka-Pronicka K, Pollak A, Skórka A, Lechowicz U, Korniszewski L, Westfal P, Skarżyński H. Płoski R Audio-profiles in mitochondrial deafness m.1555A $>\mathrm{G}$ and $\mathrm{m} .3243 \mathrm{~A}>\mathrm{G}$ show distinct differences. Med. Sci Monit 2015 Mar 6;21:694-700. doi: 10.12659/MSM.890965.

14. Mangabeira Albernaz PL. Hearing loss, dizzness and carbohydrate metabolism. Int Arch Otolaryngolog. 2016;20:261-70.

15. Ciorba A, Chicca M, Bianchini C, Aimoni C. Pastore A Sensorineural hearing loss and endothelial dysfunction due to oxidative stress: Is there a connection? J Internat Adv Otol. 2012;8(1):16-20.

16. Prasad KN, Bondy SC. MicroRNAs in Hearing Disorders: Their Regulation by Oxidative Stress, Inflammation and Antioxidants Front. Cell Neurosci. 2017;11:276. doi:10.3389/fncel.2017.00276.

17. Kondo T, Saigo S, Ugawa S, Kato M, Yoshikawa Y, Miyoshi N, Tanabe K. Prebiotic effect of fructooligosaccarides on the inner ear of DBA/2J mice with early-onset progressive hearing loss $\mathrm{J}$ Nutrition Bioch 2020;75 doi:10.1016/j.nutbio.2019.108247.

18. Hanisch F, Rahne T, Plontke SK. Prevalence of hearing loss in patients with late-onset Pompe disease: Audiological and ontological consequences. Int J Audiol. 2013 Dec;52(12):816-23. Doi:10.3109/14992027.2013.840932.

19. Szklanny K, Gubrynowicz R, Iwanicka-Pronicka K, Tylki-Szymanska. A Analysis of voice quality in patients with late-onset Pompe disease Orphanet J Rare Dis 2016 Jul 15;11(1):99. doi: 10.1186/s13023-016-0480-5.

20. Musumeci O, Catalano N, Barca E, Ravaglia S, Fiumara A, Gangemi G, Rodolico C, Sorge G, Vita G, Galletti F. Toscano A.Auditory system involvement in late onset Pompe disease: a study of 20 Italian patients. Mol Genet Metab. 2012 Nov;107(3):480-4. doi:10.1016/j.ymgme.2012.07.024. Epub 2012 Aug.

21. Sol Kang YK, Kang JA, Lee DH, Kim. Jung Sub Lim A case of autosomal dominant osteopetrosis type 2 with a CLCN7 gene mutation. J Clin Res Pediatr Endocrinol. 2019;11(4):439-44.

22. Zemojtel T, Köhler S, Mackenroth L, Jäger M, Hecht J, Krawitz P, Graul-Neumann L, Doelken S, Ehmke N, Spielmann M, Oien NC, Schweiger MR, Krüger U, Frommer G, Fischer B, Kornak U, Flöttmann R, Ardeshirdavani A, Moreau Y, Lewis SE, Haendel M, Smedley D, Horn D, Mundlos S, Robinson PN. Effective diagnosis of genetic disease by computational phenotype analysis of the diseaseassociated genome. Sci Transl Med. 2014;3(252):252ra123. 6 ) . 
23. Trubicka J, Filipek I, Iwanowski P, Rydzanicz M, Grajkowska W, Piekutowska-Abramczuk D, Chrzanowska K, Karkucińska-Więckowska A, Iwanicka-Pronicka K, Pronicki M, Łastowska M, Płoski R, Dembowska-Bagińska B. Constitutional mosaicism of a de novo TP53 mutation in a patient with bilateral choroid plexus carcinoma. Cancer Genet. 2017 Oct;216-217:79-85. doi:

10.1016/j.cancergen.2017.07.001. Epub 2017 Jul 20.

\section{Figures}
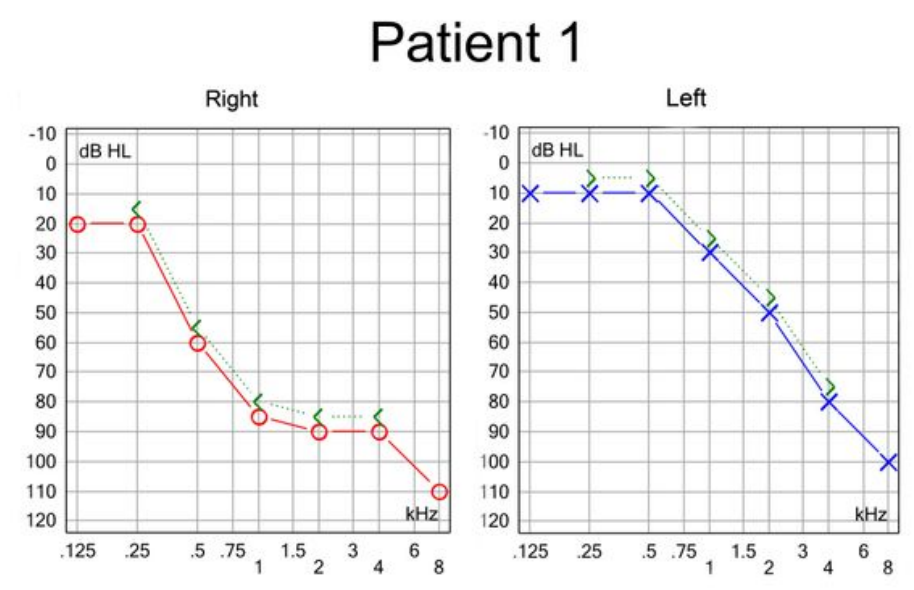

Patient 3
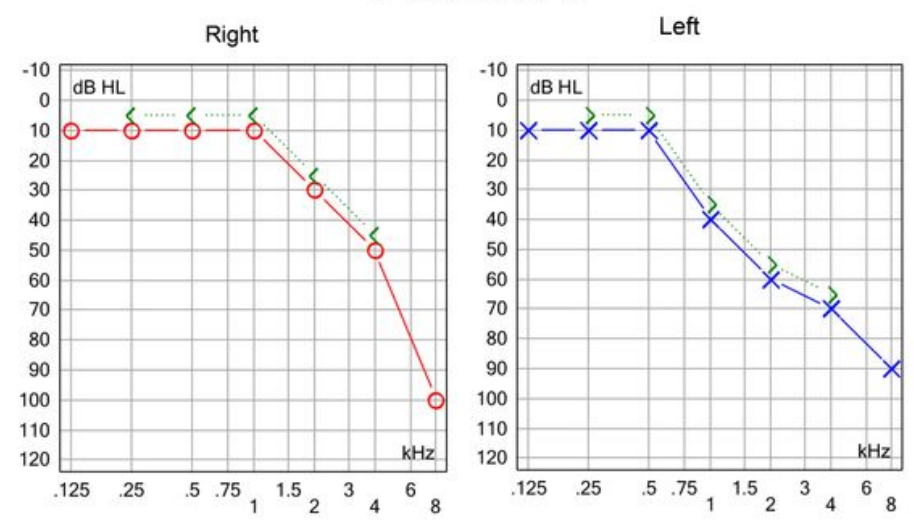

Patient 2

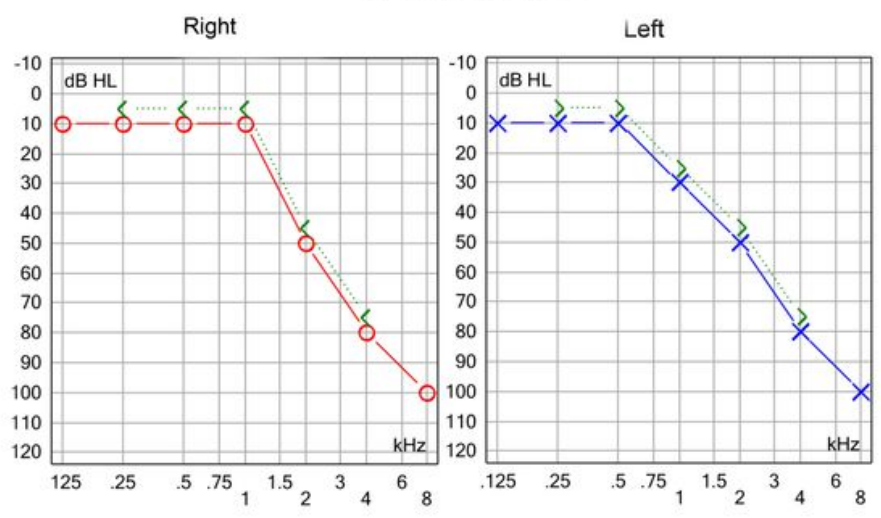

Patient 4

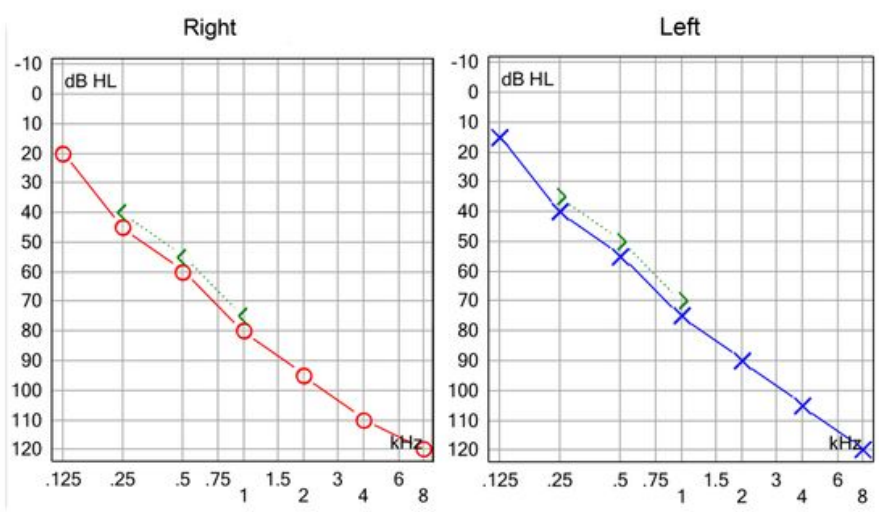

\section{Figure 1}

Audiograms of GSDI patients with hearing impairment 


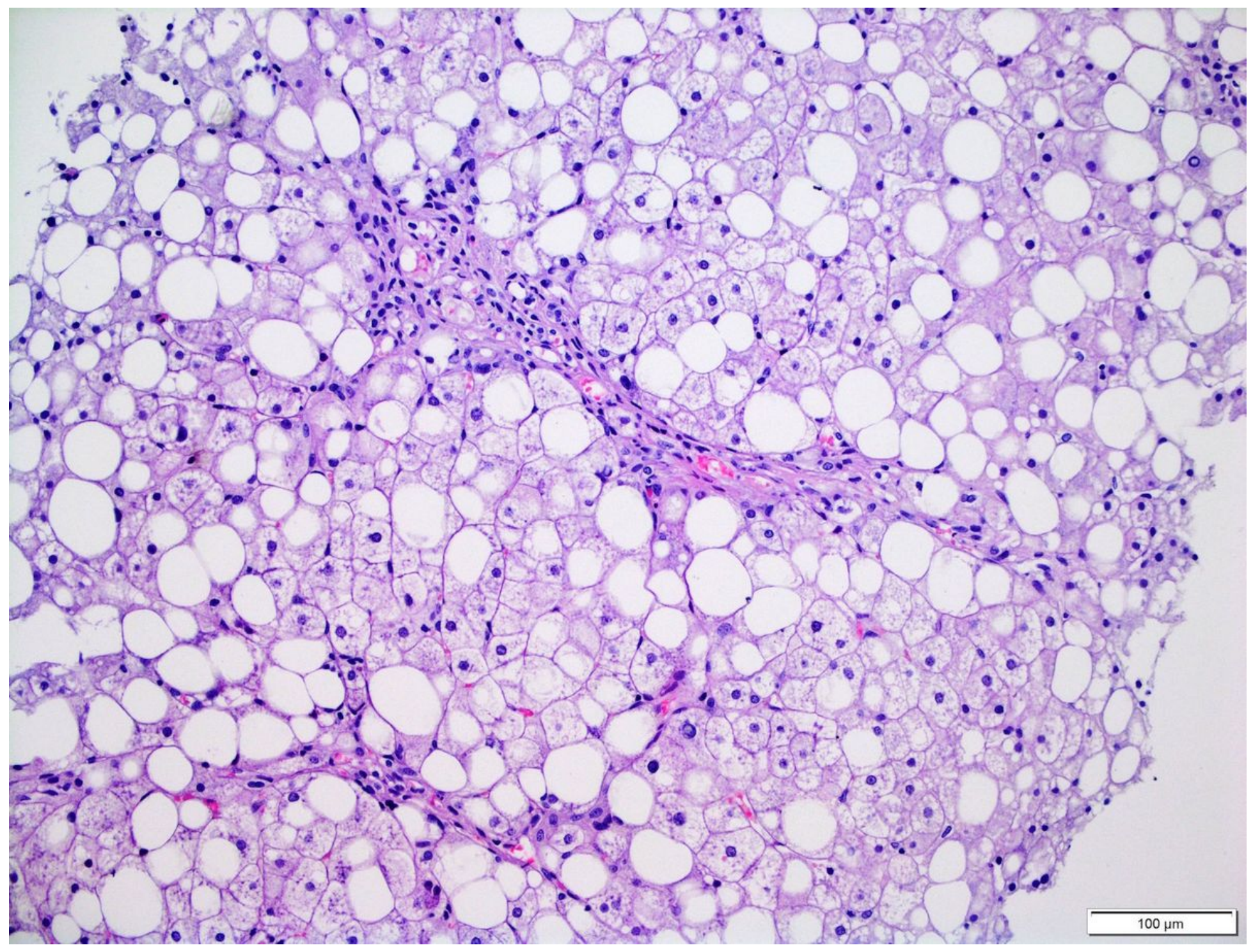

Figure 2

Liver steatosis. Hamatoxylin and eosin stain, original magnification 200X. 


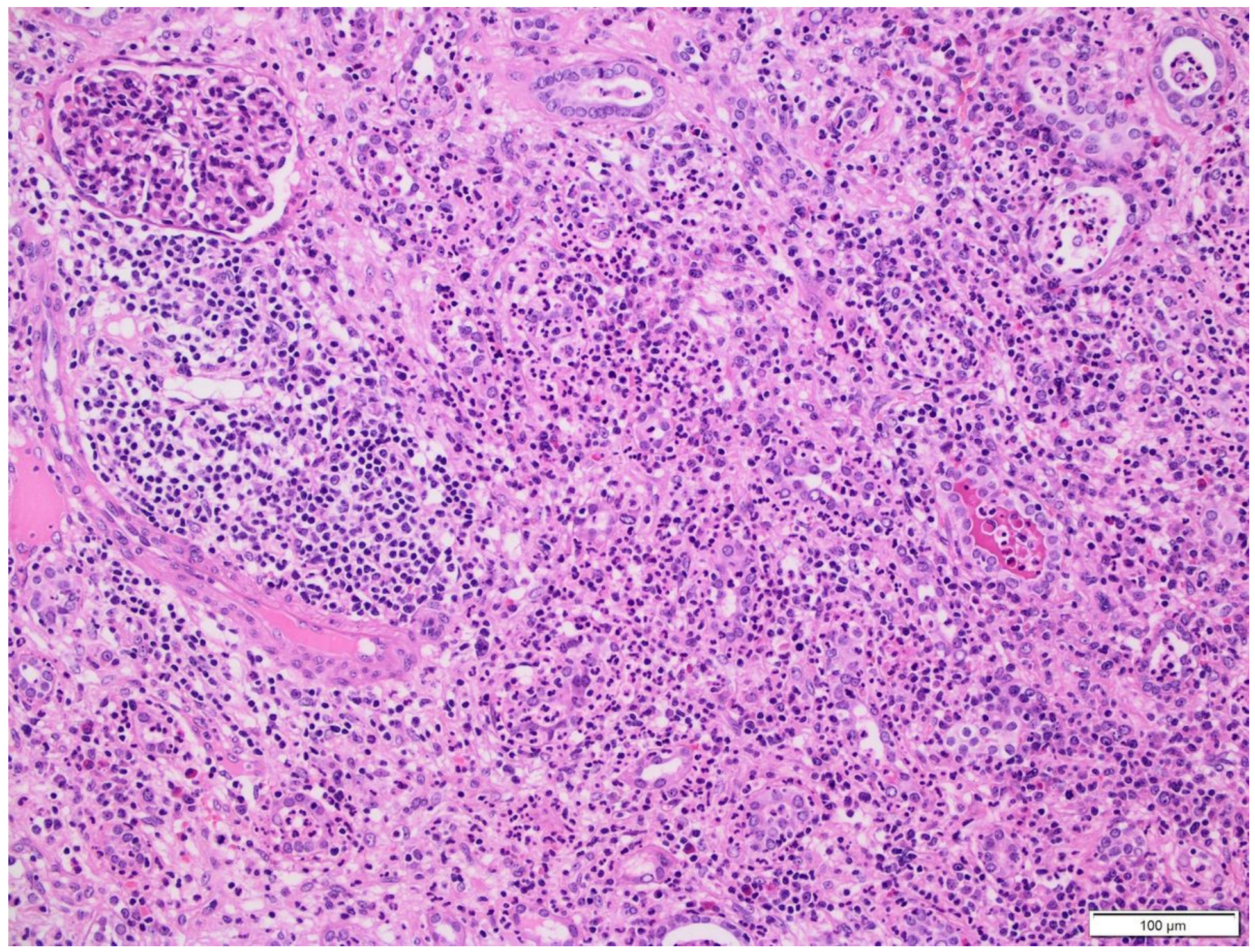

Figure 3

Interstitial renal inflammation. Hamatoxylin and eosin stain, original magnification 200X. 